\title{
DESENVOLVIMENTO E SOBREVIVÊNCIA NINFAL DE PODISUS NIGRISPINUS (DALLAS) E SUPPUTIUS CINCTICEPS (STÅL) (HETEROPTERA, PENTATOMIDAE) EM DIFERENTES TEMPERATURAS
}

\author{
Julcemar Didonet ${ }^{1}$ \\ José Cola Zanuncio ${ }^{2}$ \\ Carlos S. Sediyama ${ }^{3}$ \\ Marcelo C. Picanço $^{2}$
}

\begin{abstract}
Nymphal. DEVElopMent OF PODISUS NIGRISPINIS (DALl.AS) AND

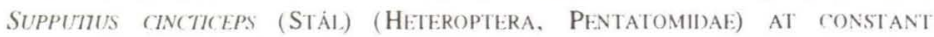
TEMPI:RATt Res. The effect of temperature on the development of Podisus nigrispints (Dallas, 1851) and Supputius cincticeps (Stảl, 1860) was studied in order to adjust equations to prediet the development of these predators under 18, 20, 25. 30, 32 and $35^{\circ} \pm 0.2^{\circ} \mathrm{C}$, relative humidity of $65.10 \%$ and photophase of 12 hours. Incubation period of the eggs and duration and viability of nymphal stage were quadratically influenced by temperature while egg viability decreased linearly with increased temperature. The best temperature for egg development was $29^{\circ} \mathrm{C}$ for both species and for nymphal development of $P$. nigrispints and $S$. cincticeps they were $29^{\circ}$ and $28^{\circ} \mathrm{C}$, respectivelly. Nymphal viahility was highly affected by extreme temperatures. with optima around $24^{\circ} \mathrm{C}$ for $S$. cincticeps and $26^{\circ} \mathrm{C}$ for $P$. nigrispinus.
\end{abstract}

KEY WORDS. Heteroptera, Pentatomidae, Podisus nigrispinus, Supputius cincticeps, nymphal development, predators

Os insetos são afetados pelas condições climáticas, sendo a temperatura um dos fatores mais importantes que influenciam no comportamento, distribuição e abundância de suas espécies (MESSENGER 1959).

Segundo CHAMPLAin \& ButTLER (1967), o efeito da temperatura é importante para a determinação do ambiente ótimo de criação massal de inimigos naturais e também para prever a duração dos estágios imaturos em temperaturas conhecidas. Modelos fenológicos, baseados na relação entre temperatura e taxa de desenvolvimento, podem ser usados para prever a ocorrência de insetos, no campo, e a melhor época para o seu controle (LISYK \& NEALIS 1988). O desenvolvimento de sistemas planejados para o uso de predadores, em programas de manejo integrado de pragas, depende da compreensão das relações entre temperatura e desenvolvimento dessas espécies (DE CleRCQ \& DEGHEELE 1992).

1) Universidade de Tocantins, 77410-470 Gurupi. Tocantins, Brasil.

2) Departamento de Biologia Animal. Universidade Federal de Viçosa. 36571-000 Vięosa. Minas Gerais, Brasil.

3) Departamento de Fitoteenia, Universidade Federal de Viçosa. 36571-000 Viçosa, Minas Gerais, Brasil. 
Este trabalho objetivou verificar o efeito da temperatura na taxa de desenvolvimento e na sobrevivência dos estágios imaturos de Podisus nigrispimus (Dallas, 1851) $(=$ P. connexivus Bergroth, 1891) e Supputius cincticeps (Stål, 1860) (Heteroptera, Pentatomidae).

\section{MATERIAL E MÉTODOS}

O experimento foi conduzido no laboratório de Entomologia Florestal do Departamento de Biologgia Animal da Universidade Federal de Viçosa (Viçosa, Minas Gerais). Os insetos foram criados em câmaras climatizadas, em seis temperaturas constantes: $18,20,25,30,32$ e $35 \pm 0,2^{\circ} \mathrm{C}$, fotofase 12 luz por 12 escuro e $65 \pm 10 \%$ de umidade relativa do ar. Os ovos de $P$. nigrispinus e $S$. cincticeps foram provenientes da criação massal em laboratório, constantemente renovada com material coletado em plantios de eucalipto. Cem ovos de cada espécie foram coletados e mantidos em cinco placas de Petri de plástico $(9,0 \mathrm{~cm}$ de diâmetro por $1,5 \mathrm{~cm}$ de altura), cada uma delas contendo 20 ovos e um chumaço de algodão umedecido, para a manutenção da umidade. Foram realizadas observações diárias para veriticar o início da eclosão, após a qual, as ninfas foram individualizadas em copos plásticos de $250 \mathrm{ml}$, segundo metodologia proposta por ZANUNCIO et al. (1992a), com algodão umedecido no fundo do copo, eliminado quando da passagem para o segundo estádio. A alimentação dos predadores, após este estádio, foi à hase de larvas de Tenebrio molitor Linnaeus, 1758 (Coleoptera: Tenebrionidae) criadas em bandejas plásticas com farelo de trigo e $5 \%$ de levedura (ZAMPERLini et al. 1992). Foram feitas observaçōes diárias para o fornecimento de alimento, ág̨ua e verificação da mudança de estádio, mortalidade e eclosão das ninfas. Foram analisados: o período de incubação, a percentagem de eclosão, a duração e a viabilidade de cada estádio e de toda a fase ninfal.

O delineamento experimental foi o inteiramente casualizado, com quinze repetições, sendo a unidade experimental constituída por quatro ninfas.

Efetuou-se a análise de variância para cada espécie, isoladamente, utilizando o Sistema de Análises Estatisticas e Genéticas (S.A.E.G.), desenvolvido pelo Centro de Processamento de Dados da Universidade Federal de Viçosa. Foram ajustadas equações de regressão, em função da temperatura, com base na significância dos componentes da equação e na representatividade do fenômeno biológico.

\section{RESULTADOS E DISCUSSÃO}

\section{Periodo de INCUBAção}

Ocorreu decréscimo quadrático e significativo do período de incubação em temperaturas de $18^{\circ} \mathrm{C}$ a $29^{\circ} \mathrm{C}$, em uma relação inversa entre a temperatura e o período de incubação (Tabs I, II). A temperatura de $29^{\circ} \mathrm{C}$ pode ser considerada como ótima, para as duas espécies de percevejos predadores, pois propiciou o máximo de desenvolvimento embrionário, com um mínimo de duração do período de incubação (Tah. II). Resultado semelhante foi obtido por BUTLLER JR. (1966), 
que observando o desenvolvimento de várias espécies de percevejos predadores, obteve períodos decrescentes de desenvolvimento embrionário com a elevação da temperatura até $30^{\circ} \mathrm{C}$. O efeito foi letal à $35^{\circ} \mathrm{C}$, exceto para Orius tristicolor (White, 1879) (Heteroptera, Anthocoridae).

Tabela I. Período de incubação (PI) e viabilidade (V|AB) dos ovos de Podisus nigrispinus e Supputius cincticeps em diferentes temperaturas, à umidade relativa do ar de $65 \pm 10 \% \mathrm{e}$ fotofase de 12 horas. Viçosa, Minas Gerais (1993).

\begin{tabular}{cccccc}
\hline \multirow{2}{*}{$\begin{array}{c}\text { Temperatura } \\
\left({ }^{\circ} \mathrm{C}\right)\end{array}$} & \multicolumn{2}{c}{ P. nigrispinus } & & \multicolumn{2}{c}{ S. cincticeps } \\
\cline { 2 - 3 } \cline { 5 - 6 } & PI (dias) & VIAB (\%) & & PI (dias) & VIAB (\%) \\
\hline 18 & 9,8 & 91,0 & & 10,8 & 68,0 \\
20 & 6,8 & 90,0 & & 7,7 & 66,0 \\
25 & 3,8 & 90,0 & & 5,1 & 65,0 \\
30 & 3,2 & 71,0 & & 3,8 & 67,0 \\
32 & 3,3 & 78,2 & & - & - \\
\hline CV $(\%)$ & 7,2 & 11,9 & & 8,9 & 17,7 \\
\hline
\end{tabular}

(-). Não houve eclosão de ninfas.

Tabela II. Equações de regressão, coeficientes de determinação $\left(R^{2}\right)$ e temperaturas ótimas (TO) da duração das fases de ovo $(Y)$ e ninfa $\left(Y^{\prime}\right)$ de Podisus nigrispinus e Supputius cincticeps em função da temperatura. Viçosa, Minas Gerais (1993).

\begin{tabular}{|c|c|c|c|c|c|c|}
\hline \multirow{2}{*}{ Fase } & \multicolumn{3}{|l|}{$P$. nigrispinus } & \multicolumn{3}{|l|}{ S. cincticeps } \\
\hline & Equaçāo & $R^{2}(\%)$ & TO $\left(^{\circ} \mathrm{C}\right)$ & Equação & $R^{2}(\%)$ & TO $\left({ }^{\circ} \mathrm{C}\right)$ \\
\hline Ovo & $Y=50,6767 \cdot 3,3364 \mathrm{~T}+0,0582 \mathrm{~T}^{2}$ & 96,9 & 29 & $Y=49,1318-3,1106 \mathrm{~T}+0,0534 \mathrm{~T}^{2}$ & 94,0 & 29 \\
\hline Ninfa I & $Y^{\prime}=36,6865-2,4373 T+0,0433 T^{\prime}$ & 76,4 & 28 & $Y^{\prime}=34,2471-2,1577 T-0,0373 T^{\prime}$ & 88,7 & 28 \\
\hline Ninfa II & $Y^{\prime}=38,8913 \cdot 2,3572 \mathrm{~T}+0,0385 \mathrm{~T}^{2}$ & 92,6 & 30 & $Y^{\prime}=54,8080-3,6029 T+0,0373 T^{\prime}$ & 90.8 & 28 \\
\hline Ninfa III & $Y^{\prime}=43,4682 \cdot 2,8462 \mathrm{~T} \cdot 0,0497 \mathrm{~T}^{\prime}$ & 87,2 & 28 & $Y^{\prime}=58,0801-4,0207 T \cdot 0,0741 T^{\prime}$ & 85.4 & 27 \\
\hline Ninfa IV & $Y^{\prime} \cdot 48,0892-3,1893 \mathrm{~T} \cdot 0,0559 \mathrm{~T}^{\prime}$ & 89,3 & 28 & $Y^{\prime}=55,7103 \cdot 3,7772 T+0,0685 T^{\prime}$ & 87,9 & 27 \\
\hline Ninfa $V$ & $Y^{\prime}=56,4970 \cdot 3,5705 \mathrm{~T} \cdot 0,0603 \mathrm{~T}^{\prime}$ & 92,4 & 29 & $Y^{\prime}-72,6290-4,8143 \mathrm{~T}=0,0851 \mathrm{~T}^{\prime}$ & 95,1 & 28 \\
\hline Ninfal & $Y^{\prime}=223,6790-14,4046 \mathrm{~T}, 0,2480 \mathrm{~T}^{2}$ & 93,8 & 29 & $Y^{\prime}=275,5650-18,3808+0,3286 \mathrm{~T}^{\prime}$ & 97,1 & 28 \\
\hline
\end{tabular}

ISENHOUR \& YEARGAN (1981), trabalhando com o percevejo predador Orius insidiosus (Say, 1831) (Heteroptera, Anthocoridae) também obtiveram resultados semelhantes, com a média da duração decrescendo, significativamente, com a temperatura, sendo de 8,8 dias à $20^{\circ} \mathrm{C}$ e de 3,5 dias a $32^{\circ} \mathrm{C}$. BRAMAN et al. (1984), verificando o efeito da temperatura no desenvolvimento e sobrevivência de Nabis americoferus (Carayon, 1961) e Nahis roseipennis (Reuter, 1873) (Heteroptera, Nabidae), também obtiveram dados semelhantes para as duas espécies. Nas temperaturas extremas, 15 e $33^{\circ} \mathrm{C}$, o período de incubação foi de 31,4 e 5,8 e de 35,3 e 8,4 dias para $N$. americoferus e $N$. roseipennis, respectivamente. As duas espécies completaram o desenvolvimento nesta faixa de temperatura. 
No presente trabalho, a temperatura de $35^{\circ} \mathrm{C}$ foi letal para $P$. nigrispinus, enquanto que para $S$. cincticeps, $32^{\circ} \mathrm{C}$ foram suficientes para que não se registrassem eclosões nesta espécie, indicando que o limite térmico superior está próximo destas temperaturas (Tab. I). Houve, diferença significativa, entre as duas espécies quanto à viabilidade dos ovos, em relação à temperatura. De $18^{\circ}$ a $30^{\circ} \mathrm{C}$, para $S$. cincticeps, o período de incubação, foi diferente, nas várias temperaturas, mas não alterou a viabilidade que a $18^{\circ} \mathrm{C}$ pode ser considerada alta para as duas espécies. ZANUNCIO et al. (1992b) relataram menor viabilidade de ovos de P. nigrispinus a $25^{\circ} \mathrm{C}$, que as obtidas neste experimento.

\section{FASE NINFAL}

As ninfas das duas espécies completaram o desenvolvimento entre $18 \mathrm{e}$ $30^{\circ} \mathrm{C}$. No entanto, somente $P$. nigrispinus completou o desenvolvimento a $32^{\circ} \mathrm{C}$ (Tab. III). Com o aumento da temperatura, o tempo requerido para completar o desenvolvimento, em todos os estádios, decresceu até $29^{\circ} \mathrm{C}$ para $P$. nigrispinus e até $28^{\circ} \mathrm{C}$ para $S$. cincticeps, podendo assim considerarem-se estas temperaturas como ótimas, para o desenvolvimento ninfal destas espécies (Tab. II).

Tabela III. Duração (dias) e viabilidade (\%) da fase ninfal de Podisus nigrispinus e Supputius cincticeps em diferentes temperaturas, à umidade relativa do ar de $65 \pm 10 \%$ e fotofase de 12 horas. Viçosa, Minas Gerais (1993).

\begin{tabular}{cccccc}
\hline \multirow{2}{*}{$\begin{array}{c}\text { Temperatura } \\
\left({ }^{\circ} \mathrm{C}\right)\end{array}$} & \multicolumn{2}{c}{ P. nigrispinus } & & \multicolumn{2}{c}{ S. cincticeps } \\
\cline { 2 - 3 } \cline { 5 - 6 } & Duração (dias) & Viabilidade (\%) & & Duração (dias) & Viabilidade (\%) \\
\hline 18 & 47,6 & 21,6 & & 52,2 & 21,6 \\
20 & 30,2 & 58,3 & & 37,6 & 58,3 \\
25 & 20,4 & 85,0 & & 22,4 & 85,0 \\
30 & 15,9 & 76,6 & & 19,6 & 28,3 \\
32 & 15,8 & 46,6 & & - & - \\
\hline CV $(\%)$ & 5,7 & 29,0 & & 6,7 & 40,2 \\
\hline
\end{tabular}

(-). Não houve eclosão de ninfas.

Em temperaturas mais baixas, o ciclo das duas espécies foi mais longo. A $18^{\circ} \mathrm{C}$ a atividade metabólica possivelmente decresceu, resultando em maior tempo para completar o ciclo de vida. Entre as temperaturas mínima e ótima de desenvolvimento tem-se uma elevação da taxa de crescimento populacional, possivelmente devido as altas temperaturas fornecerem mais energia para as reações metabólicas (METZLER 1979; Higley et al. 1986).

Os valores ótimos de temperatura, para a viabilidade, foram menores que o ótimo de temperatura para a duração da fase ninfal (Tabs III, IV). A mortalidade foi afetada pelos extremos de temperatura, sendo mais acentuada a $18^{\circ} \mathrm{C}$. $\mathrm{Na}$ temperatura intermediária de $25^{\circ} \mathrm{C}$, a sobrevivência ninfal foi de $85 \%$ para as duas espécies, valores esses mais elevados que os observados por ZANUNCIO et al. (1992b), que obtiveram índice de sobrevivência de 68,8\%. DE ClerCQ \& DEGHEELE (1992) também verificaram aumento da mortalidade nos valores extremos de temperatura e alta sobrevivência em valores intermediários. 
Tabela IV. Equações de regressão, coeficientes de determinação $\left(R^{2}\right)$ e temperaturas ótimas (TO) da viabilidade acumulada das fases de ovo ( $Y$ ) e ninfa ( $Y^{\prime}$ ) de Podisus nigrispinus e Supputius cincticeps em função da temperatura. Viçosa, Minas Gerais (1993).

\begin{tabular}{|c|c|c|c|c|c|c|}
\hline \multirow{2}{*}{ Fase } & \multicolumn{3}{|l|}{ P. nigrispinus } & \multicolumn{3}{|l|}{ S. cincticeps } \\
\hline & Equaçāo & $R^{\prime}(\%)$ & TO $\left.1^{\circ} \mathrm{C}\right)$ & Equação & $R^{2}(\%)$ & TO $\left({ }^{\circ} \mathrm{C}\right)$ \\
\hline Ovo & $Y=113,2570-1,1527 T$ & 27,7 & & - & . & . \\
\hline Ninfa II & $Y^{\prime}=181,4850 \cdot 20,0808 \mathrm{~T}-0,3649 \mathrm{~T}^{2}$ & 47,2 & 27 & $Y^{\prime}=462,0870+47,3652 \mathrm{~T}-1,0122 \mathrm{~T}^{\prime}$ & 42,8 & 23 \\
\hline Ninfa III & $Y^{\prime}=395,7020+36,6700 \mathrm{~T}-0,6882 \mathrm{~T}^{2}$ & 46,1 & 26 & $Y^{\prime}=689,4710+64,8303 \mathrm{~T}-1,3471 \mathrm{~T}^{2}$ & 52,1 & 24 \\
\hline Ninfa IV & $Y^{\prime}=524,2440+47,3189 \mathrm{~T}-0,9105 \mathrm{~T}^{2}$ & 56,8 & 26 & $Y^{\prime}=799,8000+74,0580 \mathrm{~T}-1,5451 \mathrm{~T}^{2}$ & 58,6 & 24 \\
\hline Ninfa $V$ & $Y^{\prime}=630,7560+55,8314 \mathrm{~T}-1,0806 \mathrm{~T}^{2}$ & 63,6 & 25 & $Y^{\prime}=857,6910+78,4506 \mathrm{~T}-1,6304 \mathrm{~T}^{2}$ & 60.8 & 24 \\
\hline
\end{tabular}

Considerando-se a influência da temperatura na duração da fase ninfal e na sobrevivência dos insetos, na faixa de temperatura estudada infere-se que, em surtos de insetos-praga, pode-se manejar a temperatura da criação massal de predadores para a obtenção mais rápida de maior número de indivíduos predadores. Pode-se inferir, também, que na introdução de insetos predadores no campo, é importante verificar as condições climáticas da região, em especial a temperatura, que pode favorecer ou prejudicar o estabelecimento desses agentes de controle biológico. Em condições mais favoráveis, o desenvolvimento é mais rápido e pode-se ter maior eficiência na redução populacional dos insetos-praga.

AGRADECIMENTOS. Ao BIOAGRO, CAPES, CNPq \& FAPEMIG, pelas bolsas e auxílios concedidos. Ao Programa Cooperativo de Manejo Integrado de Pragas Florestais (PC-MIP), da Sociedade de Investigações Florestais (SIF), pelo apoio na realização desta pesquisa.

\section{REFERÊNCIAS BIBLIOGRÁFICAS}

Braman, S.K.; P.E. Sloder Beck \& K.V. Yeargan. 1984. Effects of temperature on the development and survival of Nabis americoferus and $N$. roseipennis (Hemiptera: Nabidae). Ann. Ent. Soc. Amer. 77 (5): 592-596.

Butller JR., G.D. 1966. Development of several predaceous Hemiptera in relation to temperature. J. Econ. Ent. 59 (5): 1307-1307.

Champlain, R.A. \& G.D. Buttler. 1967. Temperature effects on development of the egg and nymphal stages of Lygus hesperus (Hemiptera: Miridae). Ann. Ent. Soc. Amer. 60 (3): 519-521.

De ClercQ, P. \& D. Degheele. 1992. Development and survival of Podisus maculiventris (Say) and Podisus sagitta (Fab.) (Heteroptera: Pentatomidae) at various constant temperatures. Can. Ent. 124 (1): 125-133.

Higley, L.G.; L.P. Pedigo \& K.R. Ostlie. 1986. DEGDAY: A program for calculation degree-days, and assumptions behind the degree-day approach. Environm. Ent. 15 (55): 999-1016.

ISENHOUR, D.J. \& K.V. YEARGAN. 1981. Effect of temperature on the development of Orius insidiosus with notes on laboratory rearing. Ann. Ent. Soc. Amer. 74 (1): 14-116. 
LYSYK, T.I. \& V.G. NEALIS. 1988. Temperature requeriments for development of the jack pine budworm (Lepidoptera: Tortricidae) and two of its parasitoids (Hymenoptera). J. Econ. Ent. 81 (4): 1045-1051.

Messenger, P. S. 1959. Bioclimatic studies with insects. Annu. Rev. Ent. 4: 183-206.

METZLER, D.E. 1979. Biochemistry: The chemical reaction of living cells. New York, Academic Press, 237p.

Zamperlini, B.; J.C. Zanuncio; J.E.M. Leite \& M.A.L. BraganÇA. 1992. Influência da alimentação de Tenebrio molitor (Coleoptera: Tenebrionidae) no desenvolvimento ninfal de Podisus connexivus Bergroth, 1891 (Hemiptera: Pentatomidae). Rev. Árv. 16 (2): 224-230.

Zanuncio, J.C.; J.B. Alves; R.C. Sartório \& J.E.M. Leite. 1992a. Métodos para criação de hemípteros predadores de lagartas. An. Soc. Ent. Bras. 21 (2): 245-251.

Zanuncio, J.C.; E.C. Nascimento; G.P. Santos \& F.S. Araúuo. 1992b. Aspectos biológicos do percevejo predador Podisus connexivus (Heteroptera: Pentatomidae). An. Soc. Ent. Bras. 20 (2): 243-249.

Recebido em 13.VII.1994; aceito em 20.IX.1995. 